\title{
PUTTING ROMAN DAMS IN CONTEXT: A VIRTUAL APPROACH
}

\author{
M. J. Decker, J. P. Du Vernay, J. B. Mcleod \\ Center for Virtualization and Applied Spatial Technologies (CVAST), University of South Florida, Tampa (mjdecker@usf.edu; \\ jduverna@usf.edu; jbmcleod@usf.edu
}

Commission \#\#\#\#\#\#

KEY WORDS: Terrestrial Laser Scanning, Drone-based Photogrammetry, Point Cloud, 3D Modeling, GIS, Roman Dam, Spain

\begin{abstract}
:
Water resources and management have become a critical global issue. During the half-millennium of its existence, the Roman Empire developed numerous strategies to cope with water management, from large-scale urban aqueduct systems, to industrial-scale water mills designed to cope with feeding growing city populations. Roman engineers encountered, adopted, and adapted indigenous hydraulic systems, and left lasting imprints on the landscape of the Mediterranean and temperate Western Europe by employing a range of water technologies. A recent academic study has enabled the identification of remains of and references to seventy-two dams from the Roman era, constructed in Spain between the 1st and 4th century AD. Such unique heritage, without comparisons in the Mediterranean makes Spain an emblematic case study for the analysis of Roman hydraulic engineering and water management policies. Fifty dams have been located and detailed. The twenty-two outstanding, although identified on the ground, have not been able to be acceptably characterized, due in some cases to their being ruins in a highly degraded state, others due to their being masked by repairs and reconstructions subsequent to the Roman era. A good example of such neglected dams is the buttress dam of Consuegra, in Toledo province (Castilla-La Mancha). Dating to the 3rd - 4th century AD, the Dam of Consuegra, on the basin of the Guadiana, with its over 600 metres length and 4,80 metres height, is a remarkable case of Roman engineering mastery. It had a retaining wall upstream, numerous buttresses and perhaps an embankment downstream, of which no remains are left. The application of 3D digital imaging technique to create a high quality virtual model of such monuments has proved to be successful especially for the study of the technological aspects related its construction. The case study of the Roman dam of Muel (Zaragoza) has shown, in fact, as best practices in digital archaeology can provide an original and innovative perspective on a long time studied monument. In this paper it will be explored how deploying recent computer technologies to the Roman dam at Consuegra can advance our understanding of the history of local and regional landscape change and the technology of water management. In summer 2016, the dam has been documented with terrestrial laser scanning with two FARO Focus 3D x330 and aerial photogrammetry image capturing with a DJI Phantom 4 drone. Data was processed in various 3D software applications to generate 3D representations of the dam including 3D point clouds, animations, and meshed models.
\end{abstract}

\section{INTRODUCTION}

\subsection{Project Introduction and Scope}

In 2016, the University of South Florida's Center for Virtualization and Applied Spatial Technologies (CVAST) initiated a 3D documentation project at multiple archaeological and cultural heritage sites in the Castilla-La Mancha region of Spain. The project has focused on the implementation and integration of various documentation and mapping technologies including terrestrial and close-range 3D scanning, aerial and ground-based photogrammetry as well as high resolution and panoramic photography. To date, 22 sites in Castilla-La Mancha region have been digitally documented by CVAST to varying degrees as part of this project. The objective of this paper is to detail the preliminary results for one of these Castilla-La Mancha cultural heritage sites 3D documented by CVAST, the Roman Dam of Consuegra (Figure 1).

The format of this paper is as follows: first a brief historical introduction to the Roman dam of Consuegra is presented. This historical background is followed by a discussion of the $3 \mathrm{D}$ documentation methods used for the project. Next, the 3D data processing workflow is presented and is followed by a presentation of project results. The primary objective of this project was to produce a digital record and map of the dam using multiple 3D technologies and to use these collected datasets to generate various virtual representations (i.e., 3D models, orthographic imagery, point cloud visualizations). Additionally, the integration of the collected 3D data with other datasets, including publically available aerial LiDAR, was an important aspect of our virtual approach. Here, this data integration was realized in a GIS environment, providing a wider geographic and topographic perspective of the Roman Dam.

\subsection{Roman Dam of Consuegra, Castilla-La Mancha, Spain}

During the Roman era of the Iberian Peninsula (ca 218 B.C.E.ca. 400 C.E.) local communities constructed numerous barrages on permanent and seasonal watercourses throughout the region. Today Spain and Portugal possess the most surviving Roman era dams, with the remains of more than seventy such structures currently known. Roman hydraulic engineers, while often quite advanced, were typically restricted to secondary watercourses; in part this is why they have survived in such numbers.

The dam of Consuegra is one such barrage. This structure was built on a seasonal watercourse in the basin of the Guadiana River in the current Toledo province of Castilla-La Mancha. In the 1980’s several scholars (García-Diego et al 1980; García-Diego and Fernández, Casado 1983a; García-Diego and Fernández, Casado 1983b; García-Diego and Fernández, Casado 1983c) described the Consuegra dam architecturally and dated its construction to the second century C.E. While the Consuegra barrage survives to a height of $4.8 \mathrm{~m}$, its extant length of over $600 \mathrm{~m}$ makes it one of the longest surviving dams of Roman construction. Fourteen stone buttresses survive in the middle of the dam and these were supplemented by an earthen embankment 


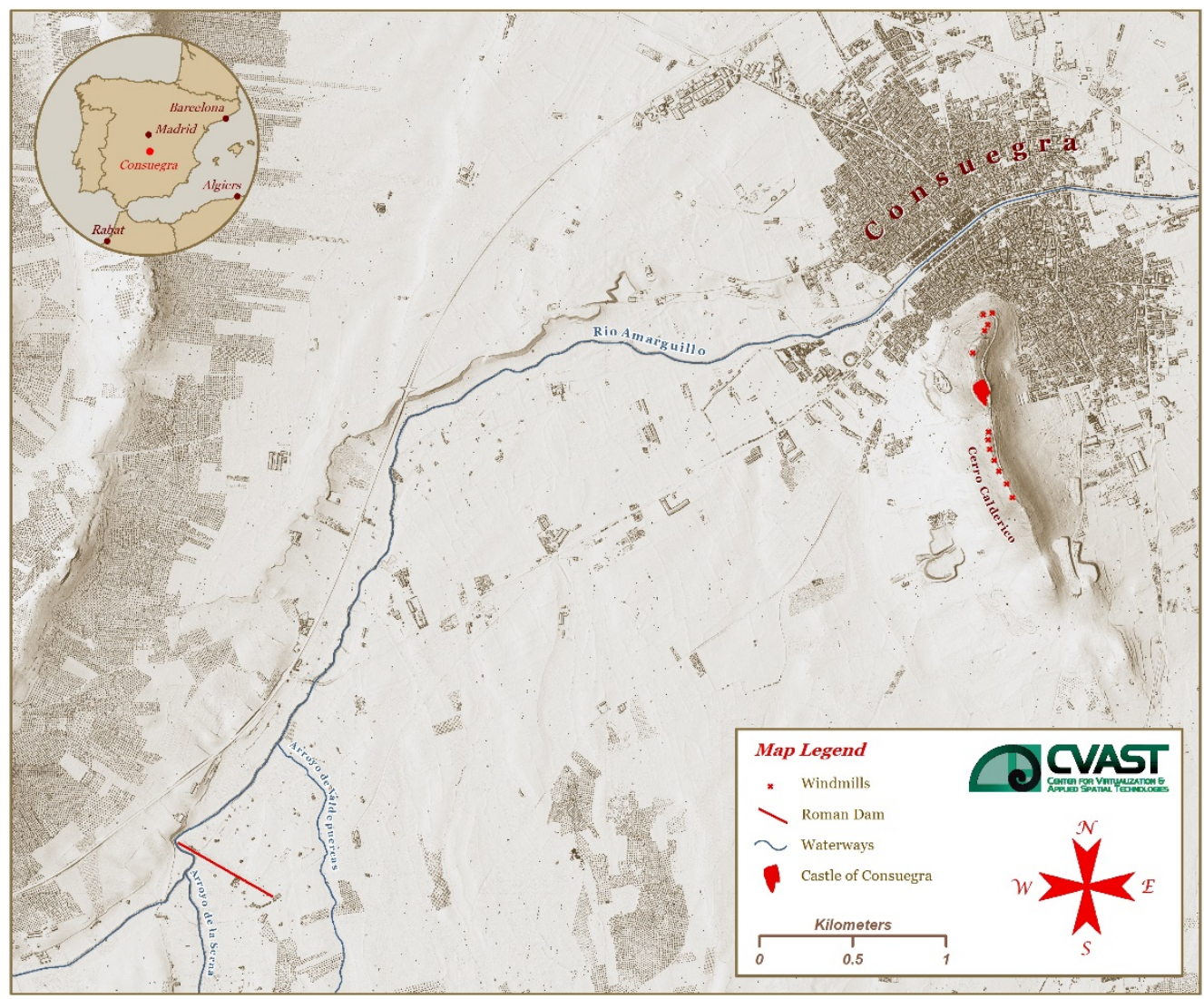

Figure 1. Map showing location of Roman Dam, related water features and Castillo de Consuegra.

which does not survive. The Consuegra dam functioned as part of a scheme of flood control and seasonal irrigation to the rich croplands around the Roman city of Consabura (today Consuegra). At least portions of the dam were in use until 1891 when a major failure led to the flooding of Consuegra. The longevity of use, the size of the structure, and the state of preservation of the Consuegra dam makes it an ideal case study for the application of current technologies in order to understand its phasing and nature of construction.

\section{DATA COLLECTION METHODS}

As recently discussed by Galeazzi (2017: 159), there has been notable increase in the incorporation of 3D technologies in the documentation and recording of archaeological and cultural heritage sites on a global scale (e.g.,Aveta et al. 2017; Chapman et al. 2013; Fiorillo et al. 2013; Lambers et al. 2007). This increase can be attributed to both the recognized archaeological research value these technologies have as tools for enhancing our knowledge of the past but also to the progressive decrease in cost and increase in user accessibility these technologies (and associated software applications) have undergone. Additionally, such technologies have become increasingly utilized in this research domain because of their recognized efficiency in data collection but also for the improved spatial data accuracy and resolution potential they offer over the more traditional spatial documentation and measurement recording methods. The resultant 3D datasets also have broad application possibilities not possible before, allowing researchers, students, and the general public to experience ancient (and sometimes not so ancient) sites in new and exciting ways.

The technologies utilized to 3D capture the Roman dam of Consuegra include terrestrial laser scanning (TLS) and dronebased photogrammetry. As discussed in the section above, the dam extends approximately 600 meters in a northwest-southeast direction. Project time constraints limited our data collection timeframe to approximately three and a half hours; as such, it was not possible to 3D document the entire dam using TLS. The entire dam length, however, was subjected to multiple drone flights, with imagery of it captured along its entire extent from multiple perspectives (discussed below).

\subsection{TLS Data Collection}

A small, approximately 45 meter section of the dam was selected for TLS, located approximately 355 meters and 175 meters inward from the northwest and southeast ends of the dam, respectively (Figure 2, left). This section of the dam was specifically targeted for TLS because it contained an interior, subterranean area that could not be captured using the drone (Figure 2, right). There are various terrestrial laser scanners available on the market with differing degrees of accuracy and resolution potential as well as methods of data collection (i.e., time of flight, phase-shift). Here, as well as at other sites included as part of the broader CVAST Castilla-La Mancha 3D documentation project, the FARO Focus X-series terrestrial laser scanner was utilized, including two FARO Focus X330 scanners and one FARO Focus X120. These scanners have an accuracy of $+/$ - $2 \mathrm{~mm}$ for a single scan. 

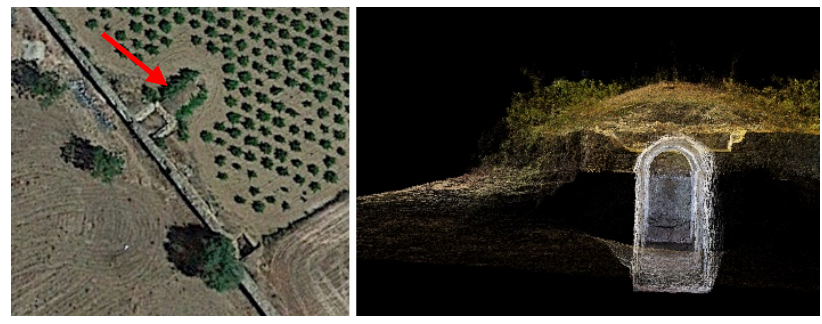

Figure 2. Area of Roman Dam captured with TLS (left), red arrow indicating location of subterranean structure; front view, point cloud render of subterranean structure (right)

The FARO Focus X-series comprises a suite of phase-shift terrestrial laser scanners, with the capability of collecting coordinate data at a distance of 330 meters. These tripod-based scanners are line of sight instruments, meaning they only can capture point data on what is visible from a scan given position. As such, it is often required to move scanner(s) around a given environment in order to capture the desired 3D data. Additionally, the FARO Focus X-series is capable of capturing coordinate data at different resolution and quality settings; these settings directly impact the point density and noise-level of the resultant point cloud dataset, but also the total time it takes to complete a single scan. For the Roman dam of Consuegra, the three FARO Focus scanners used were set to collect data at 1/4 resolution with a $4 \mathrm{x}$ quality setting selected. At this setting, the FARO scanners collect 244,000 points per second. Additionally, the FARO X-series scanners are manufactured with an integrated, high resolution color camera, permitting the collected individual points (represented in terms of their intensity value) to be assigned a RGB value as well. Although not directly relevant to coordinate data collection, the color imagery available with the scanner allows the collected point cloud data to be visualized in a more fully representational manner. At the resolution and quality setting selected and with the integrated color camera enabled, each scan position for the project took 10 minutes and 12 seconds to complete.

During the terrestrial laser scanner data collection, all three FARO Focus X-series scanners were used in tandem, and a total of 25 scan positions were completed. Scan positions were done on both sides of the dam wall and several were completed with the scanner placed on top of the wall itself (Figure 3). To facilitate the registration or stitching together of the 25 different scan positions, white spherical reference targets were placed throughout the scanning environment; targets, organized in groups of three or four, were kept within a distance of no more than $15 \mathrm{~m}$ from associated scan positions; the average distance between scan positions completed was no more than $30 \mathrm{~m}$. These distances ensured that there would be a sufficient number of points on the spherical targets to make them useful as registration objects, and that the point density of the data collected would be sufficient enough for a robust point cloud representation of the dam.

\subsection{Drone-based Photogrammetry Data Collection}

As mentioned above, the 3D documentation of the Roman dam of Consuegra also included drone-based photogrammetry image capturing. The drone utilized for the project was a DJI Phantom 4. This drone is equipped with a GPS system and a 12.4 megapixel digital camera affixed to a three axis gimbal. Multiple flight paths were flown over the dam, including a nadir oriented path flown from end to end and directly over the top of the dam as well as two oblique oriented flight paths, flown along both sides of the dam. Flight elevations ranged between 20 to $30 \mathrm{~m}$ above the ground surface and flight patterns were established using the Pix4D app. Images were captured in RAW format and a color checker was used to calibrate and control for lighting/color.

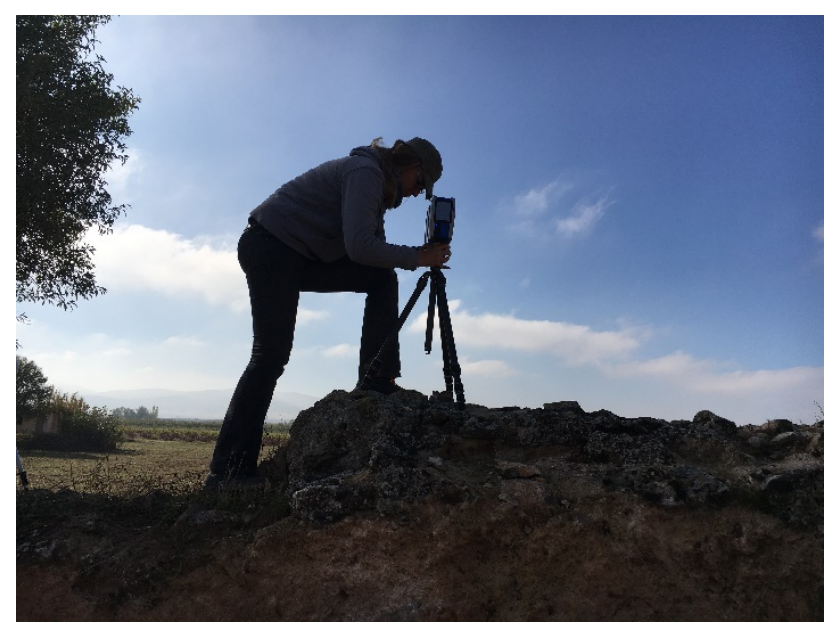

Figure 3. TLS data collection on top of section of Roman dam

\section{DATA PROCESSING}

The collected datasets were processed and integrated using multiple software applications; processing included scan data registration, filtering/editing, decimation, and exporting, photogrammetric photo alignment, 3D mesh generation, and GIS integration.

\subsection{TLS Data Processing}

The initial alignment of the TLS data was performed in FARO's proprietary registration software SCENE 5.5.3. Except in special circumstances, all CVAST scanning projects utilize the external reference objects or spherical targets (discussed above) in the scanning workflow in order to expedite the registration of the project's scans. SCENE has several options for aligning individual scans including an automatic reference target algorithm that automates the registration process. The automatic registration process identifies reference spheres of a known dimension, and in the case of this project, $145 \mathrm{~mm}$ and $139 \mathrm{~mm}$ spheres were used. The sphere fitting algorithm attempts to match constellations of the reference spheres common to two or more scans, thus aligning a sequence of scans into a single unified coordinate system and point cloud.

Being that this was a relatively small scan project (i.e., 25 total scan positions), the automatic registration process produced an acceptable registration result, and no additional, manual registration processes were necessary (Figure 4). Subsequent to alignment, the scans points were colorized based on images collected by the scanner's integrated camera, and a SCENE Project Point Cloud was created. The Project Point Cloud is FARO SCENE's version of a fast point rendering spatial data structure (i.e., Octree Database). While FARO SCENE in most cases is a powerful and intuitive scan registration software the additional point cloud processing functions are limited to point cloud filtering and cleaning, export, and orthographic image generation. There is, however, an ever expanding app center that can provide additional functionally to the stand-alone software 


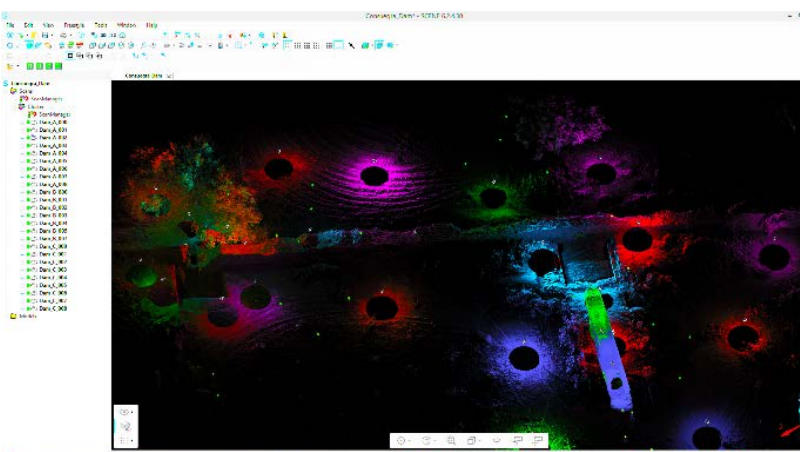

Figure 4. Screen capture showing registered TLS point cloud of Roman Dam in SCENE 5.5.3

package. The final process performed in SCENE was the exporting of the registered point cloud in .pts and .E57 formats for further processing in third-party software platforms.

The scan data exported in .pts format were imported into the free, open-source point cloud processing software CloudCompare for additional cleaning and editing. The primary use of CloudCompare was to prepare these data for integration with aerial LiDAR data in a GIS environment. The workflow to prepare these data included manually sectioning the Roman dam feature from the surrounding captured terrain and then filtering and subsampling the data to an appropriate spatial resolution for use in a GIS. The terrain data was filtered for ground using the Hidden Point Removal (HPR) plugin and the Label Connected Components tool in CloudCompare.

The HPR plugin is a point cloud filtering algorithm that effectively removes points not visible in the viewport (Katz et al. 2007). The workflow for using the HPR tool to filter for ground points involves orienting the point cloud in a bottom view thus making only the ground points visible to the viewport 3D camera and changing the viewport to a viewer-based perspective. A viewer-based perspective is required to enable and use the HPR plugin. For this project the HPR filter was applied at an Octree level of 10 which resulted in a large amount of undesirable data to be filtered out. The remaining data above the desired ground surface were largely removed using the Label Connected Components segmentation tool found in CloudCompare. This tool segments a selected point cloud into a number of components or smaller clouds based on a defined minimum distance between components (Figure 5). The minimum distance can be controlled by increasing or decreasing the octree level, and the component properties can be controlled by defining the minimum number of points required to make a component. After the filter was applied to this project's data, the undesirable components were deleted leaving a relatively clean terrain surface for use in a GIS environment. The final process for preparing the terrain data was to subsample the data to a spatial resolution of $10 \mathrm{~cm}$ and export as a las file type.

The dam feature required a manual approach to cleaning and preparation for application in a GIS environment. After cleaning the dam, the data were subsampled to a spatial resolution of 5 $\mathrm{mm}$. The difference is sampling was to allow the data to be used in GIS without loss of resolution for the dam feature.

Polygonal modeling of the Roman TLS data utilized a RealityCapture workflow, requiring an .E57 file format export from SCENE. RealityCapture is a photogrammetric processing software that aligns photos and scans to produce high-resolution

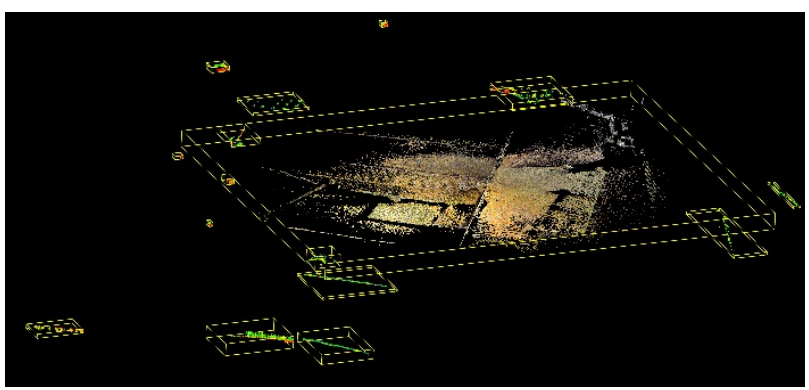

Figure 5. Labeled Connected Components segmentation tool in CloudCompare

textured mesh models. Processing the Roman dam in RealityCapture involved importing the .E57 file from SCENE and aligning the scans in RealtyCapture which produces a point cloud. The point cloud is then processed into a mesh that was textured based on the RGB values collected by the laser scanners' integrated cameras. The textured model was then exported from RealityCapture in .obj format and brought into Meshlab for final cleaning, repair polygonal decimation, and image rendering. The model generated was composed of 13 million faces making it too large for use in a GIS. This being the case, the Quadric Edge Collapse Decimation (with texture) filter in the Meshlab was used to decimate the model to 277,000 faces which is an acceptable size for a GIS environment. The final step for preparing the model for use in a GIS was to export the model from Meshlab as a Collada (DAE) file type.

\subsection{Drone-based Photogrammetry Data Processing}

Additionally, as discussed above, the entire dam was captured using a DJI Phantom 4 drone. The drone images were aligned and processed in Pix4D using a standard image processing workflow. A 3D textured mesh of the dam was generated in the software with a total polygonal count of one million triangles. Additionally, for this project, the point cloud produced from the processing of the nadir images provided the most useful data for a GIS integration and analysis as it allowed for a higher resolution DEM when combined with the aerial LiDAR data (results discussed below).

\subsection{Geographic Information System (GIS) Data Processing}

As detailed above, an objective of the project was to integrate the collected 3D data in an online GIS environment but also to utilize publically available aerial LiDAR data to contextualize the Roman Dam in its wider geographic and topographic context. To this end, aerial LiDAR data from the Centro Nacional de Información Geográfica was obtained.

Aerial LiDAR for the City of Consuegra and areas west to include the Rio Amarguillo and the Roman Dam were downloaded. The average point spacing for the dataset is 1.133 meters, and it covers an expansive area of 48 square $\mathrm{km}$. As it will be detailed below, these data were used to generate both a Digital Surface Model (DSM), and a Hillshade Surface Model. The aerial LiDAR points were filtered for ground, vegetation and structures. The DSM was computed using a Natural Neighbor interpolation method at 0.5 meter resolution. The resulting digital surface model was used to generate the aforementioned Hillshade Surface Model. 
The decimated (277,000 faces), textured meshed model of the Roman Dam made from the TLS data was integrated into the GIS environment. The model (in Collada, .DAE format) was imported into a Geodatabase (.gdb) as a textured multi-patch feature class and was georeferenced in the UTM ETRS 1989 ETRS-TM30 coordinate system, the same as the aerial LiDAR obtained from Centro Nacional de Información Geográfica. To georeference the model, coincident geometry between the aerial LiDAR points and model nodes were established along the Dam structure and subterranean feature.

\section{PROJECT RESULTS AND CONCLUSIONS}

The objective of this preliminary project was to generate a 3D digital record and map of the dam but also to use the collected datasets to generate various virtual representations that included an integration into a GIS environment. Project time constraints prevented the full documentation of the dam using TLS; instead only a small, approximately 45 meter length of the dam was 3D scanned. As detailed above, the original 3D mesh from the TLS point cloud contained approximately 13 million faces; this model was decimated for various purposes, including to approximately 5 million faces to facilitate model image renders as well as 277,000 faces for GIS integration. Figure 6 illustrates a 3D model of this section of the dam that is rendered using the $\mathrm{x}$-ray shader of Meshlab; this rendering and transparent shader option was selected here to better showcase aspects of the subterranean structure as it relates to the surrounding area of the dam.

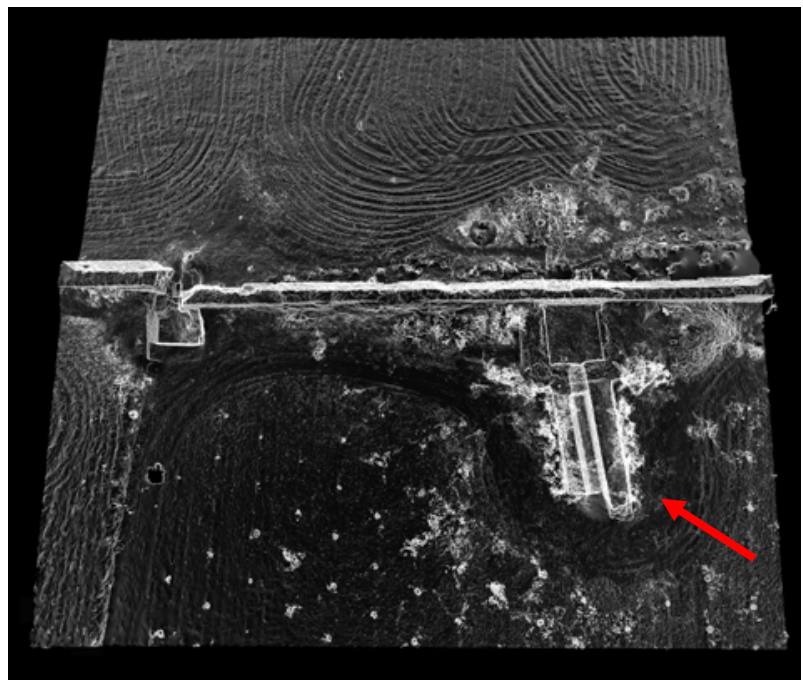

Figure 6. Render of 3D mesh of area of Roman Dam captured with TLS (Meshlab's X-ray shader applied to mesh to better show subterranean structure, red arrow)

Figure 7 illustrates an isometric view (top) and an elevation view (bottom) of the interior structure scanned with TLS. The isometric image created in CloudCompare exhibits a series of thin slices of multicolored point data extracted from the structure's point cloud model. The benefit of segmentations such as these can be used to create $2 \mathrm{D}$ images that express the $3 \mathrm{D}$ nature of an object or structure. The bottom images is an orthographic elevation that was rendered in Bentley Pointools. Orthographic images can be used in CAD programs as reference images for creating detailed drawings and creating measurements. Because these images are created from the
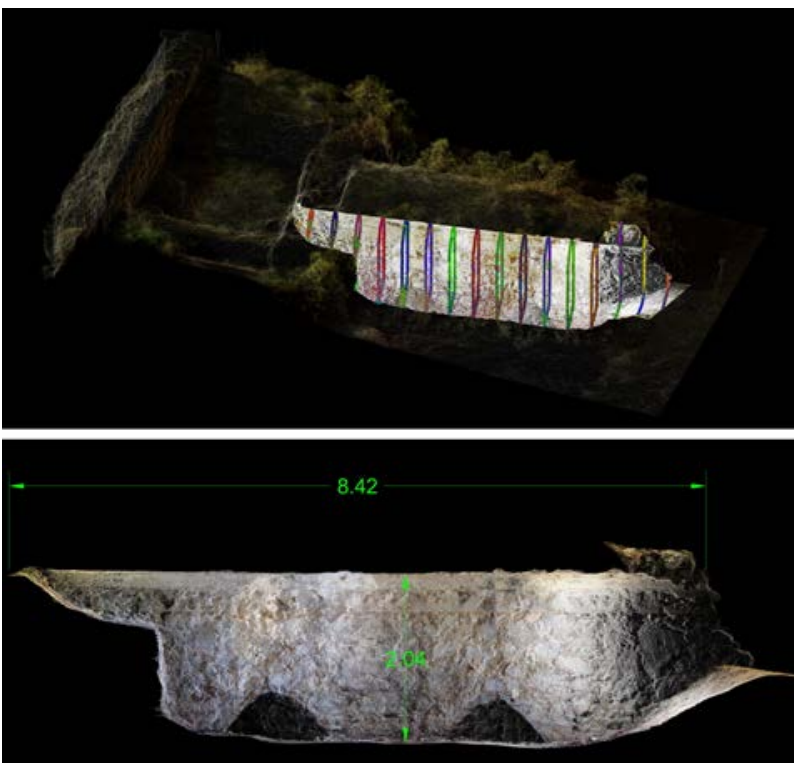

Figure 7. Point cloud renders. Isometric view of subterranean feature of Roman Dam with slices (top); orthographic elevation image of subterranean feature detailing dimension data (bottom)

coordinate data of the point cloud it is a useful way to share data information to audiences that have little to no experience with 3D information.

Although the window of opportunity to document the Roman dam was limited, the results of this project allowed us to enhance the visualization of this monument and present it within its wider geographic and topographic context on different scales. Prior to collecting the data, it was decided that an important platform would be a GIS environment and the data would be combined with publically available aerial LiDAR. This largely was decided on because of the accessibility of aerial LiDAR data and its suitability for visualizing surface models of landscape features for large spatial areas. Figure 8 displays the merging of the 3D meshed model derived from the TLS data and aerial LiDAR data. The top image displays the aerial LiDAR data processed as a DSM and merged with the 3D model; the bottom displays the aerial LiDAR data as a hillshade with the same georeferenced model. Both images help to demonstrate how the integrating of these datasets provide wider context of this monument than could be achieved with just 3D scan data alone and a higher resolution visualization of the Roman dam than could be achieved by using only the aerial LiDAR data. Similarly, the photogrammetric model derived from the drone acquisition, (a section of this mesh is displayed in Figure 9) provides another level of scale and resolution that can integrated into a GIS environment. The benefit of this approach is that it provides scholars with more comprehensive and scalable data than can be achieved using the each of the 3D technologies alone. The resulting information can then be viewed, shared, and analysed across a wide variety of visualization and analytical software platforms to facilitate research collaboration and expand research directions.

The use of advanced data acquisition and analysis techniques offers considerable promise in assisting the reconstruction of past landscapes. In specific reference to Roman water management techniques, combining 3D visualization tools and GIS allows for greater knowledge in a number of areas, namely the specifics of 
the construction and life cycle of past hydraulic systems, their wider landscape context, the utilization of such systems and why ultimately these structures failed or were abandoned.

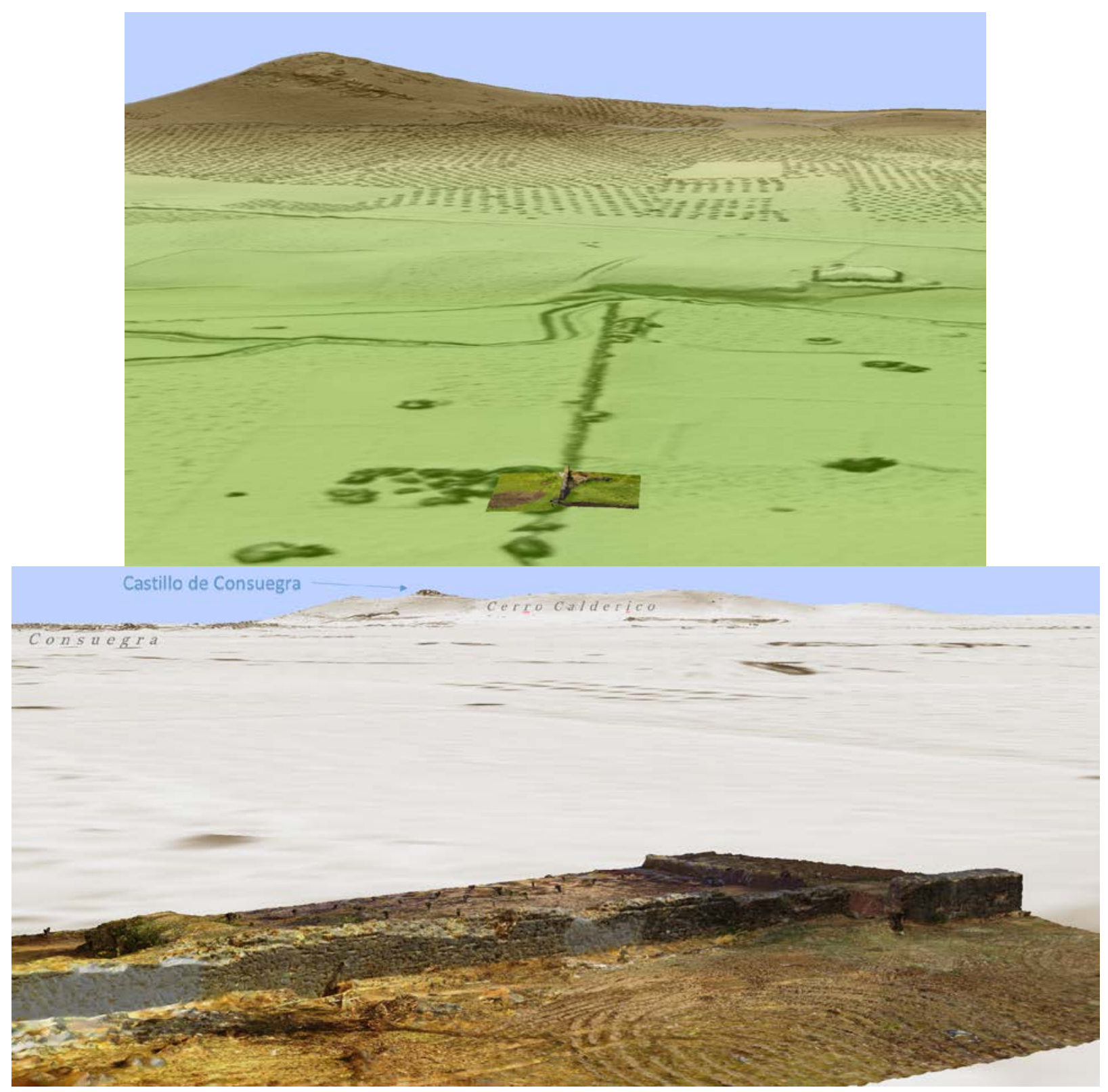

Figure 8. DSM made from aerial LiDAR data that is merged with 3D model of Roman dam made using collected TLS data (top); hillshade model made from aerial LiDAR data and same 3D model (bottom). 


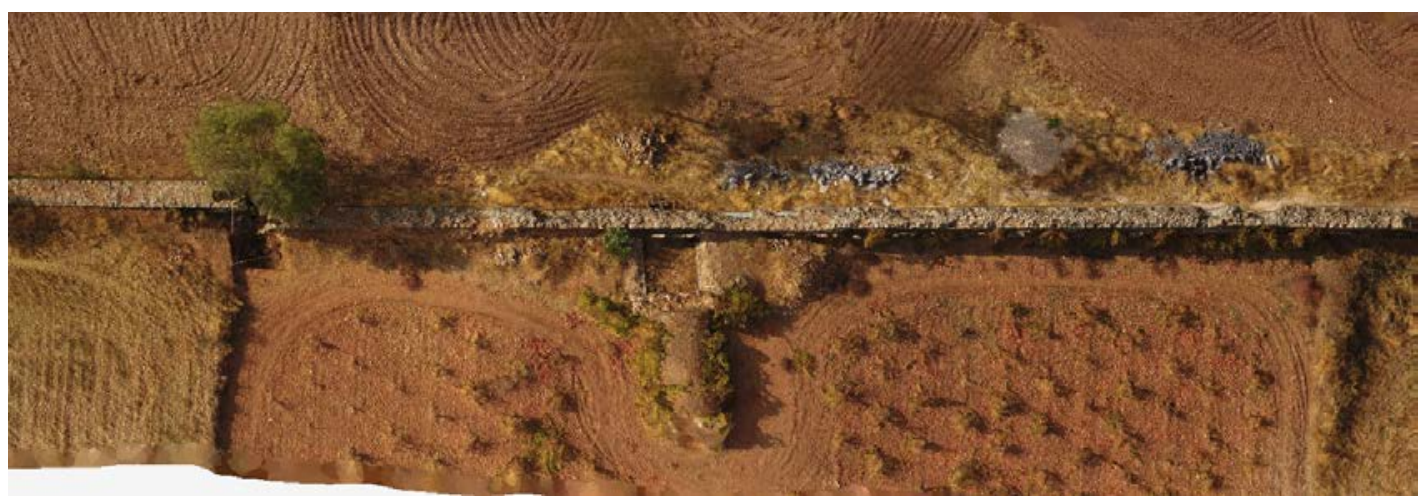

Figure 9. 3D mesh of Roman dam section generated from captured drone-based imagery

\section{ACKNOWLEDGEMENTS}

We thank Victor Manuel Lopez-Menchero Bendicho of the University of Castilla-La Mancha for coordinating our work at the Roman Dam of Consuegra and all other sites 3D documented by CVAST within the Castilla-La Mancha region; we also thank Diego Lucendo Diaz of BARAKA ARQUEOLOGOS for assisting with the drone flights for the project, and send gratitude and thanks to the Hitz Foundation for providing funding for CVAST projects.

\section{REFERENCES}

Aveta. C., M. Salvatori, G.P. Vitelli. 2017. The complex point cloud for the knowledge of the architectural heritage. Some experiences. The International Archives of The Photogrammetry, Remote Sensing, and Spatial Information Sciences Volume XLII5W/W1:235-244.

Chapman, Harry, Eamon Baldwin, Helen Moulden, and Michael Lobb 2013. More than just a sum of points: Re-thinking the value of laser scanning data. In Visual Heritage in the Digital Age, edited by Ch'ng et al., pp. 15-33. Springer Series on Cultural Computing, London.

Fiorillo, Fausta, Belen Jimenez Fernandez-Palacios, Fabio Remondino, and Salvatore Barba. 2013. 3D Surveying and modelling of the Archaeological Area of Paestum, Italy. VAR. Volume 4 (8): 55-60.

Galeazzi, Fabrizio, 2016. Toward the definition of best 3D practices in archaeology: assessing 3D documentation techniques for intra-site data recording. Journal of Cultural Heritage, pp. 159-169.

García-Diego, J., Díaz-Marta, M., Smith, N. 1980. Nuevo studio sobre la presa romana de Consuegra, Revista de obras públicas 127: 487-505.

García-Diego, J.;Fernández, Casado, C. 1983a. Estudio conjunto sobre la presa romana de Consuegra (I), Revista de obras públicas 130(3215): 491-502.

García-Diego, J.;Fernández, Casado, C. 1983b Estudio conjunto sobre la presa romana de Consuegra (II), Revista de obras públicas 130(3216): 585-99.
García-Diego, J.;Fernández, Casado, C. 1983c. Estudio conjunto sobre la presa romana de Consuegra (I), Revista de obras públicas 130(3217): 673-688.

Katz, Sagi, Ayllet Tal, Ronen Basri. 2007. Direct Visibility of Point Sets. ACM Transactions on Graphics 26(3):1-11.

Lambers K., Eisenbeiss H., Sauerbier M., Kupferschmidt D., Gaisecker T., Sotoodeh S., Hanusch T., 2007. Combining photogrammetry and laser scanning for the recording and modelling of the Late Intermediate Period site of Pinchango Alto, Palpa, Peru. Journal of Archaeological Science, 34(10), pp. 1702-1712. 